făhrt. Er lässt deswegen sein Erz direkt in einen kleinen Schachtofen eintröpfeln (Fig. 132). Ruthenburg rechnet mit der 'Tatsache, dass $\mathrm{Fe}_{3} \mathrm{O}_{4}$ bei verhaltnismåssig sehr niedriger Temperatur zu reduzieren ist. Ueber diese $\mathrm{Re}$ duktionsarbeit mit minderwertigen Gasen konnte mir Ruthenburg genaue Daten leider noch nicht angeben und sie scheinen vorläufig einen praktischen Wert noch nicht erhalten zu haben. Ruthenburg sagte mir, dass er sowohl in Kanada, als im Westen von Amerika zwei grossere Versucbsanlagen mit mehreren hundert Pferdekräften errichten werde.

Der Prozess an und für sich ist entschieden ein sehr eigenartiger und meines Wissens in deutschen Fachzeitschriften noch wenig besprochen. Man wird vorläufig aber auf Betriebsresultate noch warten mussen, bevor man ein endgültiges Urteil fällen kann. Es hat sich sčhon manches Verfahren, das im ersten Augenblick zu teuer oder zu kompliziert aussah, doch Bahn gebrochen. Mit dem Wunsche, dass es Ruthenburg gelingen möge, alle Schwierigkeiten, die ihm entgegentreten, zu überwinden und mit dem Danke an den Herrn Ruthenburg, der mir bereitwilligst alle seine Apparate und Maschinen gezeigt hat, möchte ich diese kurze Mitteilung schliessen.

Herr Professor G. Tammann-Göttingen:

UBBER GLASBILDUNG UND ENTGLASUNG.

Man teilt die verschiedenen Aggregatzustånde, in denen ein Stoff auftreten kann, am besten in die beiden Hauptgruppen isotrope und anisotrope Zustände ein.

Die isotropen Zustände sind: I. der gasförmige, 2. der flüssige und 3 . der amorphe Zustand. Diese Zustănde unterscheiden sich von dem anisotropen, dem Kristallzustande, vor allem dadurch, dass alle Eigenschaften der Stoffe in den isotropen Zuständen von der Richtung im Stoffe nicht abhängen, sie sind skalarer Natur. Dagegen ist im Kristallzustande ein Teil der Eigenschaften von der Richtung im Kristall abhăngig.

Viele Stoffe treten nun nicht nur in einer, sondern in mehreren Kristallarten auf. Der Polymorphismus ist eine sehr verbreitete Erscheinung.

Man ersieht also, dass die alte Systematik der drei Zustände: gasförmig, flüssig und fest, nicht weit reicht, wenn durch dieselbe die wesentlichen Merkmale der Zustände charakterisiert werden sollen.

Es war schon längst bekannt, dass einige Stoffe bei schneller Abkühlung ihrer Schmelzen in Form isotroper, fester Stoffe erhalten werden können. Aber man hatte sich wohl nicht genügend Rechenschaft gegeben von der Art und Weise, in der sich die spontane Kristallisation unterkühlter Schmelzen vollzieht, und deshalb hatte man auch die Bedingungen, welche erfallt sein müssen, um einen Stoff in den amorpben Zustand uberzuführen, nicht genügend erkannt.

Die spontane Kristallisation einer unterkủhlten Schmelze hăngt von zwei Faktoren ab: I. von dem spontanen Kristallisationsvermogen, das durch die Anzahl von Kristallisationscentren, die sich während der Zeiteinheit in der Masseneinheit der Flüssigkeiten bilden, gemessen wird; 2. von der Geschwindigkeit, mit der sich die
Grenze zwischen der Flüssigkeit und dem Kristall verschiebt.

\section{Spontanes Kristallisationsvermögen.}

Wir wollen uns zuerst mit dem spontanen Kristallisationsvermogen, der die Kristallisation unmittelbar bedingenden Eigenschaft, beschaftigen.

Sowohl durch Betrachtung der Struktur einer kristallisierten Schmelze, als auch durch direkte Beobachtung der Vorgänge beim Eintritt der Krystallisation kommt man zum Resultat, dass die Kristallisation auch bei grossen Unterkuhlungen nie gleichmässig durch die ganze Masse der Flüssigkeit sich vollzieht, sondern dass dieselbe immer nur in einzelnen Punkten der Flussigkeit, den Kristallisationscentren, eintritt. Von diesen aus wachsen häufig radial angeordnete Kristallfäden, durch deren Verlängerung eine Kugel oder ein Kugelsegment, ein Sphärokristall entsteht. Die Anzahl dieser Sphärokristalle, von denen jeder ein Kristallisationscentrum enthält, lässt sich bei einigen Stoffen leicht durch Zählung bestimmen.

$\mathrm{Zu}$ diesem $Z$ weck schliesst man den Stoff in cin dünnwandiges Glasrobr, von engem Lumen, erwärmt das Rohr in einem Bade auf eine bestimmte Temperatur oberhalb des Schmelzpunktes und bringt dann das Rohr schnell in ein Bad, das eine Temperatur unterhalb des Schmelzpunktes besitzt. Bis zu einer gewissen Temperatur, gegen 40 bis $80^{\circ}$ unterhalb des Schmelzpunktes, kann man dann nach einer gewissen Zeit die gebildeten Sphärokristalle, eventuell die einzelnen Kristalle, abzählen. Bei tieferen Unterkuhlungen wird die Kristallisationsgeschwindigkeit so gering, dass man zu lange warten müsste, bis die Kristallisationscentra zu sichtbaren Gebilden ausgewachsen sind. Deshalb erwarmt man den Stoff, nachdem er eine Zeit lang einer niederen Temperatur exponiert 
worden war, auf eine Temperatur, bei der die Zahl der Kristallisationscentren sehr klein ist, die Kristallisationsgeschwindigkeit aber so gross, dass nach wenigen Minuten die Zahlung vorgenommen werden kann.

Es stellte sich heraus, dass die Anzahl der Kristallisationscentren pro Zeit- und Gewichtseinheit zuerst mit steigender Unterkühlung zunimmt, dann aber bei Temperaturen um $100^{0}$ unterhab des Schmelzpunktes schnell wieder abnimmt. Die Anzahl der Kristallisationscentren, die sich in $1 / 8 \mathrm{ccm}$ Betol, Schmelzpunkt $95^{\circ}$, innerhalb zwei Minuten bei verschiedenen Temperaturen bilden, ist in Fig. I 33 dargestellt. Bei diesem Stoff ist das Maximum besonders scharf ausgeprăgt.

Man kann dieses Resultat auch noch in anderer Weise formulieren. Die Zahl der Kristallisationscentren ist ein Maass der Stabilităt

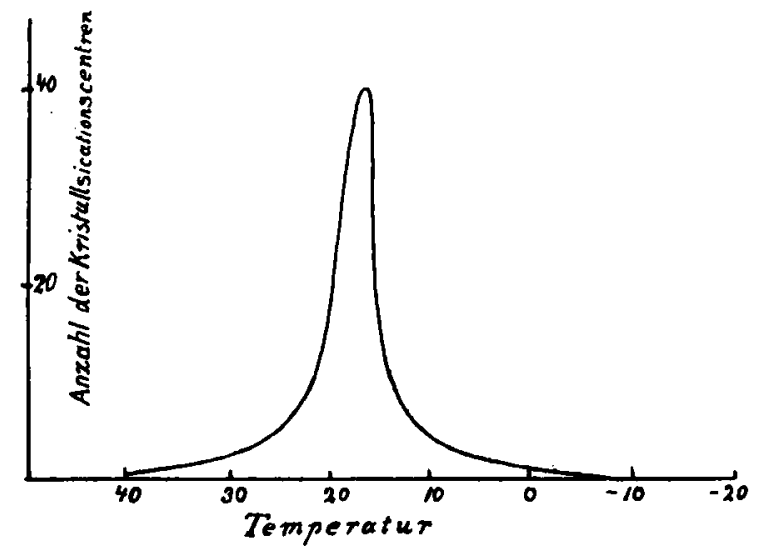

Fig. 133.

der unterkühlten Flüssigkeit, denn je grösser die Zahl der Kristallisationscentren pro Zeitund Gewichtseinheit ist, um so grösser ist auch die Neigung der unterkühlten Flüssigkeit, ihren Aggregatzustand $\mathrm{zu}$ ändern. Man kann also sagen, dass mit wachsender Unterkühlung die Stabilität der Flüssigkeit zuerst abnimmt, dann aber bei weiter wachsender Unterkühlung sehr erheblich zunimmt. Es wäre also moglich, dass tief unterhalb des Schmelzpunktes die Stabilität des amorphen Zustandes bei gewissen Stoffen grósser wird als die Stabilităt des Stoffes im anisotropen Zustande.

\section{Die Kristallisationsgeschwindigkeit.}

Gehen wir nun zur Kristallisationsgeschwindigkeit über und untersuchen deren Abhängigkeit von der Temperatur. Dazu bringt man die Schmelze in ein $\mathbf{U}$-förmig gebogenes Glasrohr, unterkablt dieselbe in einem Bade auf eine bestimmte Temperatur und berührt dann die Schmelze, nachdem sie die Temperatur des Bades angenommen hat, mit einem Kristall. Sofort beginnt die Kristallisation der unter- kăhlten Schmelze. Es wachsen bei Unterkühlungen, die grősser sind als $10^{0}$, eine Reihe von Kristallfäden, die sich alle parallel der Rohraxe ordnen, in die Schmelze. Die Enden dieser Kristallfäden, zwischen denen sich noch nicht kristallisierte Schmelze befindet, stellen sich bald in eine Ebene senkrecht zur Rohraxe ein. Die Geschwindigkeit der Fortbewegung dieser deutlich sichtbaren Grenze zwischen der klaren Schmelze und den Enden der Kristallfäden ist leicht zu messen. Es ergibt sich, dass die Abhăngigkeit der Kristallisationsgeschwindigkeit von der Temperatur des Bades, in dem sich das U-Rohr befindet, durch das Diagramm in Fig. I34 dargestellt wird, wenn der höchste Wert der Kristallisationsgeschwindigkeit über $3 \mathrm{~mm}$ beträgt.

Bei kleinen Unterkühlungen im Temperaturintervall $A$ kristallisieren flächenreiche Kristalle. Beimengungen, welche die Schmelze enthält, werden von den Kristallen nicht aufgenommen. Im Temperaturintervall $B$ wächst die Kristallisationsgeschwindigkeit, trotzdem die Temperatur des Bades abnimmt. Der Grund hierfür ist der,

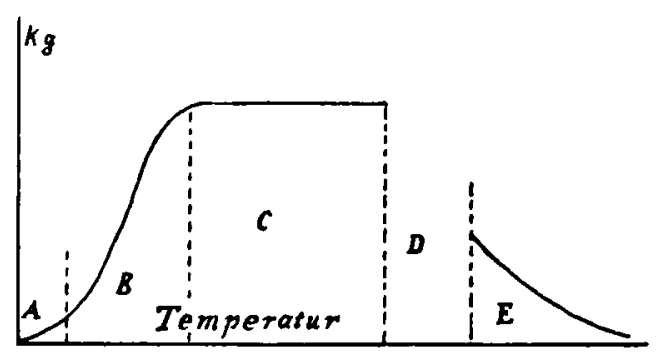

Fig. I34.

dass durch die erhebliche Kristallisationswärme auch Flüssigkeitsschichten, welche von der Kristallisationsgrenze weiter entfernt sind, bis auf die Temperatur des Schmelzpunktes erwärmt werden, dadurch wird wegen nicht genügend schnellen Warmeabflusses das Eindringen der Kristallfäden in die zu warmen Schichten verzögert, da mit sinkender Temperatur des Bades die Geschwindigkeit, mit der die Wärme abgeführt wird, wächst, so wächst auch die Kristallisationsgeschwindigkeit. Dass diese Auffassung die richtige ist, ersieht man schon daraus, dass sich die Kristallfäden in diesem Temperaturintervall nur an den Röhrenwänden bilden, der innere, zu warme Teil ist frei von denselben. Im Temperaturintervall $C$ kristallisiert an der sichtbaren Grenze gerade so viel, dass in einer dünnen Schicht an den Enden der Kristallfäden die Schmelztemperatur sich gerade herstellt. Dementsprechend ist die Kristallisationsgeschwindigkeit in diesem Fall unabhängig von der Temperatur. Die im Querschnittselement an der Kristallisationsgrenze frei werdende Wärmemenge nimmt mit sinkender Temperatur 
zu, weil im Querschnittselement mit sinkender Temperatur die Zahl der Kristallfäden und wohl auch ihre Dicke zunehmen. Schliesslich wird aber bei tiefen Unterkühlungen, auch wenn im Querschnittselement die ganze vorhandene Menge kristallisiert, die Kristallisationswărme nicht mehr genügen, um die Temperatur der Grenzschicht bis auf den Schmelzpunkt zu erwärmen. Entsprechend dieser Temperaturabnabme an der Kristallisationsgrenze, nimmt die Kristallisationsgeschwindigkeit mit wachsender Unterkühlung ab und erhält bei tieferen Unterkühlungen ausserordentlich kleine Werte.

Man kann also durch genügend schnelle $\mathrm{Ab}$ kühlung die Kristallisation einer unterkuhlten Flüssigkeit zum Stillstand bringen. Zur Demonstration dieser Hemmung der Kristallisation eignet sich dic Schmelze des Benzophenons, weil die Zahl der Kristallisationscentren im flussigen Benzophenon klein ist, die Kristallisationsgeschwindigkeit aber erheblich.

Beträgt die maximale Kristallisationsgeschwindigkeit eines Stoffes weniger als $3 \mathrm{~mm}$

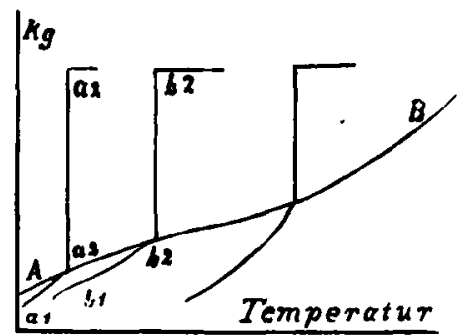

Fig. 135.

pro Minute, so reicht gewöhnlich die Wärmemenge, welche in der Zeiteinheit frei wird, nicht hin, um die Temperatur an der Kristallisationsgrenze uber ein Temperaturintervall hin konstant auf der Temperatur des Schmelzpunktes zu erhalten. Die Folge hiervon ist, dass das horizontale Stück im Temperaturintervall $C \mathbf{z u}$ einem Punkt zusammenschrumpft. Die Kurve, welche die Abhängigkeit der Kristallisationsgeschwindigkeit von der Temperatur darstellt, hat dann ein Maximum.

Bei Stoffen mit grősserer Kristallisationsgeschwindigkeit gibt es noch ein durch das besondere Verhalten der Kristallisationsgeschwindigkeit charakterisiertes Temperaturintervall $D$ (Fig. I 34), welches den Stoffen mit kleinerer Kristallisationsgeschwindigkeit fehlt. In diesem Temperaturintervall sind unter den gewöhnlichen Bedingungen der Wärmeleitung stationäre $\mathrm{Ge}-$ schwindigkeiten, welche den Badtemperaturen dieses Intervalls entsprechen, nicht realisierbar. Die an der Kristallisationsgrenze frei werdende Warmemenge ist in diesem Gebiet der Badtemperaturen zu gross, als dass unter den ge- gebenen Bedingungen der Wärmeleitung die Badtemperatur an der Kristallisationsgrenze sich dauernd erbalten könnte. Infolgedessen beobachtet man von gewissen Werten der Kristallisationsgeschwindigkeit an ein plötzliches Ansteigen derselben bis zu ihrem maximalen Wert. In dem Gebiet $D$ kann also der Kristallisationsprozess mit stetiger Beschleunigung explosionsartig verlaufen. Lässt man die Temperatur eines Bades, in dem sich ein $U$-Rohr mit einer kristallisierenden Flüssigkeit befindet, langsam steigen, so wächst die Kristallisationsgeschwindigkeit zuerst langsam mit steigender Badtemperatur, wie die Kurvenäste $a_{1} a_{2}$ und $b_{1} b_{2}($ Fig. 135) andeuten.

Je dicker das Lumen der Röhre, je grösser also die frei werdende Kristallisationswarme ist und je langsamer diesclbe abgeleitet wird, um so kleiner wird der Wert der Kristallisationsgeschwindigkeit sein, bei dem die beschlcunigte Kristallisation eintritt.

Erreicht nun bei langsam steigender Badtemperatur die Kristallisationsgeschwindigkeit einen Wert, bei dem die frei werdende Wărmemenge zu gross ist, um noch einen stationären Wert der Kristallisationsgeschwindigkeit zuzulassen, so wird die Geschwindigkeit beschleunigt, und in wenigen Sekunden wächst sie bis auf den konstanten, maximalen Wert an.

Die beiden Kurven $a_{1} a_{2} a_{2}$ und $b_{1} b_{2} b_{2}$ veranschaulichen dieses Anwachsen der Kristallisationsgeschwindigkeit, die Kurve $a_{1} a_{2} a_{2}$ bezieht sich auf eine dickere Flussigkeitssăule, die andere auf eine dunnere. Unterhalb der Kurve $A B$ sind die Geschwindigkeiten stationäre, oberhalb der Kurve $A B$ sind alle Geschwindigkeitswerte bis auf den maximalen, nicht stationär.

\section{Die Entglasung.}

Diese Verhältnisse sind für die Entglasung amorpher Stoffe wichtig. Steigert man die Temperatur eines amorphen Stoffes, so wird, wenn dieselbe ins Temperaturgebiet, in dem sich Kristallisationscentren bilden, gelangt ist, die Entglasung beginnen.

Es können sich hierbei die Kristallisationscentren verschiedener polymorpher Kristallarten bilden. Die beiden Kristallarten können nach der Entglasung bestehen bleiben, es kann aber auch vorkommen, dass schliesslich nur eine der beinen Kristallarten zuruckbleibt. Je nachdem die maximale Kristallisationsgeschwindigkeit der spontan sich bildenden Kristallart klein oder gross ist, wird sich bei der Entglasung die Temperatur der Masse durch die frei werdende Kristallisationswärme åndern. Die bei grősster Kristallisationsgeschwindigkeit wăhrend der Entglasung eintretende Temperatursteigerung $\Delta t$ wird durch die Werte der Kristallisationswärme $r$ 
und der spezifischen Wärme der sich bildenden Kristalle $c_{p}$ " bestimmt:

$$
\Delta t=\frac{r}{c_{p} \overline{ }} .
$$

Beträgt die Kristallisationswärme $50 \mathrm{~g} / \mathrm{cal}$ pro Gramm und die spezifische Warme der Kristalle $0,25 \mathrm{~g} / \mathrm{cal}$ pro Gramm, so kann die Temperatur während der Entglasung, wenn die Kristallisationsgeschwindigkeit eine grosse ist, um $200^{0}$ steigen. Beträgt die Temperatur, bei der die Bildung von Kristallisationscentren beginnt, $500^{\circ}$, so würde die Temperatur während der Entglasung auf $700^{\circ}$ steigen können, es masste dann durch die Entglasung ein lebhaftes Erglühen der Masse eintreten. Dieses Erglühen ist beim Natriummetasilikat $\mathrm{Na}_{2} \mathrm{SiO}_{3}$ leicht zu demonstrieren. Schmilzt man Soda und Kieselsäureanhydrit in äquivalenten Mengen im Platintiegel zusammen und schreckt den Tiegel in kaltem Wasser ab, so erhält man das Silikat als klares Glas, wenn die Menge des Silikates nicht mehr als $2 \mathrm{~g}$ beträgt. Erwärmt man dann den Tiegel auf etwas uber $50^{\circ}{ }^{0}$, so tritt die

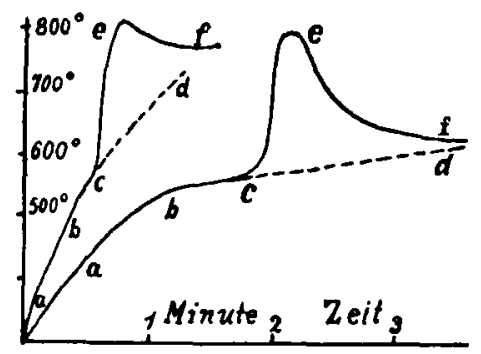

Fig. 136 .

Bildung von wenigen Kristallisationscentren ein, die Kristallisationsgeschwindigkeit wird erheblich, und bald ergluht das kristallisierende Glas.

Fig. 136 gibt die Abhängigkeit der Temperatur von der Zeit im glasigen Natriummetasilikat, wenn dasselbe mit eingeschmolzenem Thermoelement im Platintiegel uber freier Bunsenflamme erhitzt wird. Der Kurvenast $a b c$ gibt die Steigerung der Temperatur, welche durch die äussere Wärmezufuhr hervorgerufen wird; wenn keine Entglasung eingetreten wăre, so würde die Temperatur im Glase nicht über $55^{\circ}$, resp. $75^{\circ} 0^{\circ}$ auf dem Kurvenstück $c d$ gestiegen sein. Beginnt die spontane Kristallisation, so tritt ein plötzliches Steigen der Temperatur auf dem Kurvenast $c e$ ein. Je nach der Lage des Schmelzpunktes wird dann bei $e$ ein Temperaturabfall eintreten, wenn die Temperatur des Schmelzpunktes bei der Entglasung nicht erreicht wird, oder es wird sich, wenn der Schmelzpunkt erreicht wird, die Temperatur einige Zeit konstant erhalten, bis alles kristallisiert ist. Beim Natriummetasilikat, dessen Schmelzpunkt bei I055 ${ }^{\circ}$ liegt, wird die Temperatur des Schmelzpunktes nicht erreicht. Die
Kristallisationswärme des Natriummetasilikates beträgt bei $20^{0} 29$ cal pro Gramm, wie sich aus den Lösungswärmen des kristallisierten und amorphen Natriumsilikates ergab. Die spezifische Wärme der kristallisierten $\mathrm{Na}_{2} \mathrm{SiO}_{3}$ beträgt zwischen 20 und $100^{\circ} 0,197$. Es ergibt sich also aus diesen Werten, dass, wenn bei Zimmertemperatur die Entglasung momentan vor sich gehen würde, die Temperatur um $149^{\circ}$ steigen würde. Es wurde eine Steigerung um $160^{\circ}$ bei 5200 beobachtet.

Die Geschwindigkeit, mit der die Temperatur des Glases gesteigert wird, hat auf die Temperatur, bei welcher die Entglasung beginnt, keinen erheblichen Einfluss.

Die Struktur der entglasten Masse wird durch die Zahl der Kristallisationscentren und von dem Betrage der maximalen Kristallisationsgeschwindigkeit bestimmt. Ganz ahnliche Verhaltnisse hat Herr W. Gurtler bei der Entglasung des Kuprimetaborates, des Kobaltpyroborates und des Manganbiborates gefunden. Die Entglasung des amorphen Zinkmetaborates und vieler tonerdehaltigen Silikate tritt, weil hier die Zahl der Kristallisationscentren pro Zeitund Maasseinheit auch bei der Temperatur des maximalen spontanen Kristallisationsvermögens sehr gering ist, langsam ein, auch wenn das Glas die Temperatur angenommen hat, die dem Maximum des spontanen Kristallisationsvermögens entspricht. In solchen Fällen ist es zuweilen sehr zeitraubend, die Temperatur des maximalen spontanen Kristallisationsvermogens aufzusuchen. Will man aber die Entglasung sicher erzielen, so muss man über die Lage dieser Temperatur orientiert sein, da in anderen Temperaturgebieten die Zahl der Kristallisationscentren eine sehr kleine sein kann, infolgedessen also bis zum Eintritt der Entglasung ausserordentlich lange zu warten wăre.

Das spontane Kristallisationsvermogen, die Kristallisationsgeschwindigkeit und die Viskosităt.

Mit wachsender Unterkühlung nimmt die Viskosität der Flüssigkeit zu und durchlăuft häufig in einem kleinen Temperaturintervall alle Werte, von denen einer siropösen Flüssigkeit bis zu denen einer festen Masse, die durch Wirkung kleiner Krafte, welche kurze Zeit wirken, nicht deformiert wird, bei weiterer Temperaturerniedrigung wird die Masse hart und spröde.

Die Bestimmung der Viskosităt unterkahlter Schmelzen von zwölf Kohlenstoffverbindungen, deren Schmelzpunkte zwischen 80 und $180^{0}$ lagen, ergab, dass diese Flüssigkeiten ungefähr $90^{0}$ unterhalb des Schmelzpunktes ihre Beweglichkeit kleinen Kräften gegenuber verlieren.

Die Temperaturabhängigkeit der drei Eigenschaften, des spontanen Kristallisationsvermögens, 
der Kristallisationsgeschwindigkeit und der Zahhigkeit ubersieht man im Diagramm der Fig. I37. Die mit $\eta$ bezeichnete Kurve soll das Anwachsen der Viskosität mit sinkender Temperatur zur Anschauung bringen, die mit $K G$ bezeichnete Kurve gibt die Abhängigkeit der Kristallisationsgeschwindigkeit von der Temperatur an und die mit $s p . K V$ kenntlich gemachte Kurve bezieht sich auf die Abhängigkeit des spontanen Kristallisationsvermogens von der Temperatur.

Man ersieht nun, dass, wenn bei der Abkühlung das Temperaturintervall, in dem sich zablreiche Kristallisationscentren bilden, schnell durchschritten wird, die Viskosität der Flüssigkeit bald eine sehr grosse wird. Ist diese grosse Viskosität erreicht, der Stoff glasartig spröde geworden, dann bilden sich in demselben keine weiteren Kristallisationscentren, und wenn sich während der Abkuhlung einige Kristallisationscentren gebildet haben sollten, so würden dieselben doch wegen der ausserordentlich kleinen Werte der Kristallisationsgeschwindigkeit in

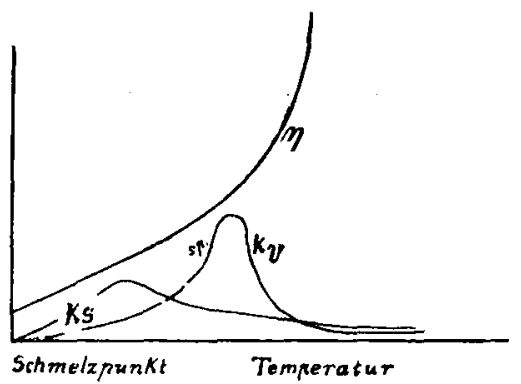

Fig. 137.

diesem Temperaturgebiet nicht weiter wachsen. Der Stoff wird also in diesem Temperaturgebiet als Glas recht stabil sein.

Die Form und die Lage der drei Kurven zueinander (Diagramm Fig. 137) ist bei verschiedenen Stoffen eine sehr verschiedene. Wenn die Viskosităt beim Schmelzpunkt schon eine grosse ist, so scheinen die maximalen Werte des spontanen Kristallisationsvermögens und der Kristallisationsgeschwindigkeit wie bei vielen tonerdehaltigen Silikaten klein zu sein.

Von der Form der Viskositätskurve und der Lage der Kurve des spontanen Kristallisationsvermögens zur Viskositätskurve wird es abhăngen, ob man einen Stoff in der Weise wie Glas zu dünnen Fäden ausziehen oder zu Gefăssen blasen kann. Eine notwendige Bedingung, die erfallt sein muss, um einen Stoff wie Glas bearbeiten zu kơnnen, ist folgende: Die Zahl der Kristallisationscentren muss im Temperaturgebiet, in dem die Viskosität die geeignete Grösse für die Bearbeitung der viskosen Flüssigkeit hat, sehr klein sein. Wichtig ist auch, dass die Viskositätskurve nicht zu steil ansteigt, da sonst das Temperaturgebiet, in dem die
Flussigkeit die zur Bearbeitung geeigneten Viskositătswerte besitzt, zu klein ist, wodurch natürlich die Bearbeitung sehr erschwert wird. In Fig. ${ }^{3} 3^{8}$ ist die Viskosităt zweier Stoffe I und 2 in Abhängigkeit von der Temperatur dargestellt. Liegen die zur Bearbeitung geeigneten Werte der Viskosität in den Grenzen $a$ und $b$, so wird das. Temperaturintervall, in dem der Stoff I bearbeitet werden kann, grösser sein, als das, in dem der Stoff 2 bearbeitet werden kann.

Für viele Zwecke wäre es wichtig, das spontane Kristallisationsvermögen beliebig vergrössern oder verkleinern zu können. Hatten wir die Aenderung der Zahl von Kristallisationscentren, die sich in unterkühlten Schmelzen bilden, in unserer Gewalt, so könnten wir nach Belieben einen Stoff als Kristallkonglomerat oder im amorphen Zustande erhalten. Wie würden wohl die Eigenschaften der Metalle bei der Ueberführung in den amorphen Zustand sich ändern? Von den Metallen ist bisher nur das Antimon als sogenanntes explosives Antimon im amorphen Zustande erhalten worden.

Tastversuche aber die Wirkung eines magnetischen oder elektrischen Feldes und der Radiumstrahlung auf die Zahl der Kristallisationscentren im unterkuhlten flüssigen Betol ergaben keinen merklichen Einfluss. Einen sehr grossen Einfluss

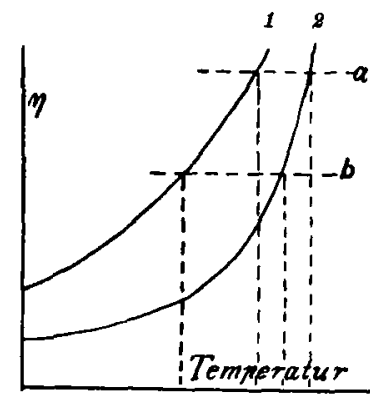

Fig. 138 . ubt aber häufig der Zusatz von Stoffen, die sich in der Schmelze auflösen, aus. Ein Teil dieser $\mathrm{Zu}$ sătze drückt die Zahl der Kristallisationscentren auch im Temperaturintervall des Maximums derselben auf Null herab, ein anderer vermehrt dieselbe stark, ohne aber die Temperatur des Maximums zu ăndern, und schliesslich wirken gewisse Zusătze in den Mengen von einigen Prozenten sehr wenig ein. Beachtenswert ist die Wirkung eines Zusatzes von indifferenten unlöslichen Stoffen, die entweder wirkungslos sind, oder aber in einigen Fallen die Zahl der Kristallisationscentren stark erhöhen oder verkleinern. Platin scheint die Zahl der Kristallisationscentren fast immer zu vermehren, von dieser Eigenschaft des Platins kann man manchmal in Fallen, wo Impfung ausgeschlossen ist, Nutzen ziehen.

\section{Können alle Stoffe in den amorphen Zustand abergefabrt werden?}

Diese Frage soll hier nur ganz einseitig beantwortet werden, indem auf das Verhalten der Stoffe bei sehr hohen Drucken nicht weiter eingegangen wird. 
Um dieser Frage in gekennzeichnetem Sinne naher zu treten, wurde eine statistische Untersuchung an ${ }^{5} 53$ Stoffen vorgenommen. Die geschmolzenen Stoffe wurden in enge, dunnwandige Glasröhren eingeführt, die Rohrchen zugeschmolzen, über die Schmelztemperatur des Stoffes erwarmt und dann schnell in Bader von verschiedenen Temperaturen unterhalb des Schmelzpunktes ubergeführt.

Von ${ }_{1} 53$ Stoffen liessen sich:

22 oder $14 \%$ um nicht mehr als $10^{0}$ einige Sekunden lang unterkühlen,

54 oder $35 \%$ um ro bis $20^{\circ}$ einige Sekunden lang unterkühlen,

I9 oder $13 \%$ um $20^{\circ}$ und mehr eine Minute lang unterkühlen und

59 oder $38 \%$ konnten im glasigen Zustand erhalten werden.

Die Geschwindigkeit der Abkühlung liesse sich jedenfalls noch bedeutend vergrössern, die Zeit, wăhrend der die unterkühlte Schmelze im Temperaturintervall grossen spontanen Kristallisationsvermoggens verweilt, also bedeutend verkürzen. Infolgedessen darf man behaupten, dass Aussicht vorhanden ist, bei schnellerer Abkühlung die Mehrzahl der Stoffe als Gläser zu erhalten.

Bei einzelnen Gruppen von Stoffen, die in obiger Zusammenstellung nicht berücksichtigt sind, ist die Zahl der Kristallisationscentren so gering, dass viele ihrer Glieder auch bei langsamer Abkuhlung leicht als Gläser erhalten werden können. So kann man viele Silikate, Metaphosphate, einige Vanadinate, manche Sulfide und Oxyde und einige nichtmetallische Elemente leicht im amorphen Zustande erhalten. Bei den Kohlenstoffverbindungen begünstigt das Vorkommen von Hydroxylgruppen, und zwar mit ihrer Anzahl in steigendem Maasse, die Unterkahlungsfähigkeit der Schmelze. Von den aromatischen Verbindungen lässt sich die Orthoverbindung tiefer als die entsprechende Paraverbindung und noch tiefer als die Orthoverbindung die Metaverbindung unterkühlen.

\section{Stabilität amorpher Stoffe.}

Bei tieferen Unterkühlungen sind die Stoffe im amorphen Zustande so stabil, dass die Entscheidung der Frage nach dem Stabilitätsverhaltnis der Stoffe im amorphen und im Kristallzustande auf Schwierigkeiten stösst. Bei direkter Berührung des Kristalls mit dem Amorphen ist ja eine Umwandlung nicht $\mathrm{zu}$ erwarten, weil die Kristallisationsgeschwindigkeit ganz ausserordentlich kleine Werte besitzt. Ferner ist die Dampfspannung, die Eigenschaft, aus deren Werten für den Kristall und das Glas am direktesten die Frage nach der Stabilität entschieden werden konnte, so klein, dass sie nicht mehr messbar ist.
Nun scheint bei derselben Temperatur der Stoff im Kristallzustande immer weniger reaktionsfahig zu sein als im amorphen Zustande. Die Losungsgeschwindigkeit des Glases ist also grösser als die des Kristalls. Die Löslichkeiten selbst kann man für beide Zustănde leider nicht gut vergleichen, da die Löslichkeit vieler amorpher Stoffe keinen bestimmten Wert besitzt, sondern eher mit der Löslichkeit quellbarer Stoffe zu vergleichen ist. In manchen Fallen tritt wăhrend der Auflösung des Glases an der Oberfläche desselben die Kristallisation ein, so z. B. beim Uebergiessen vieler amorpher Kohlenstoffverbindungen mit Alkohol oder anderen Lösungsmitteln. Auch bei den Silikaten ist die Zersetzungsgeschwindigkeit des Amorphen immer viel grösser als die des Kristalls, auf die Bedeutung dieses Unterschiedes für die Geologie hat J. Lemberg hingewiesen.

Es gibt aber Stoffe, wirkliche chemische Individuen, die nur im amorphen Zustand bekannt sind, das Borsăureanhydrid und die Metaphosphorsäure. Bei diesen Stoffen, besonders beim Borsäureanhydrid, ist wohl nicht anzunehmen, dass bei gewöhnlichem Druck eine Kristallisation aberhaupt eintreten kann, weil dieselbe ja sonst schon einmal eingetreten wäre, da das Borsäureanhydrid in ganz erheblichen Mengen produziert wird und ja auch schon sehr haufig sehr verschiedenen Temperaturen exponiert worden ist. Es drängt sich die Frage auf: Kristallisiert das Borsäureanhydrid nicht bei höheren Drucken oder kristallisiert dasselbe aberhaupt nicht?

Entsprechend der Antwort auf diese Frage, hătten wir drei Gruppen von Stoffen zu unterscheiden. I. Stoffe, bei denen das Zustandsfeld des Kristallzustandes innerhalb eines gewissen Temperaturintervalls direkt an das $\mathrm{Zu}-$ standsfeld des Dampfes grenzt; 2. Stoffe, bei denen zwischen jene beiden Zustandsfelder sich das Zustandsfeld des amorphen, resp. flüssigen Zustandes schiebt, und 3. Stoffe, die uberhaupt nicht kristallisieren. Wenn die Stoffe der beiden letzten Gruppen so wenig bekannt sind, so mag das zum Teil daran liegen, dass wir solche Stoffe nicht zu isolieren verstehen, denn die Isolierung chemischer Verbindungen grundet sich hauptsächlich auf die Kristallisation.

$$
\begin{aligned}
& \text { Volumen-und Energieinhalt des } \\
& \text { isotropen und anisotropen Zustandes. }
\end{aligned}
$$

Beim Uebergang aus dem flüssigen in den amorphen Zustand ändert sich von allen Eigenschaften nur die Viskosität ausserordentlich stark, aber nicht in diskontinuierlicher Weise. Die Verfestigung, resp. Erweichung vollzieht sich nicht bei einer bestimmten Temperatur, sondern in einem Temperaturintervall. Die anderen Eigenschaften, wie das Volumen, der 
Energieinhalt, die optischen Eigenschaften u.s.w., scheinen sich in dem Temperaturintervall, in dem sich die Viskosität so ausserordentlich ändert, nicht wesentlich anders zu ändern, als bei Temperaturen oberhalb dieses Temperaturintervalls. So ergab sich der Ausdehnungskoeffizient von Betol im Temperaturintervall von $154^{\circ}$ bis $-16^{\circ}$ als unabhăngig von der Temperatur zu $0,000265 \mathrm{ccm}$ pro Gramm, derselbe ändert sich also beim Uebergang vom flüssigen in den amorphen Zustand nicht, da das Betol bei $o^{0}$ fär kleine Kräfte nicht mehr beweglich und bei $-15^{\circ}$ fest ist.

Jedenfalls tritt beim Uebergang von einer leicht beweglichen Flussigkeit in einen amorphen festen Stoff keine diskontinuierliche Aenderung der Eigenschaften ein. In dieser Beziehung unterscheidet sich also diese Aenderung des Aggregatzustandes wesentlich von dem Uebergange in den anisotropen Zustand. Es liegen also keine Grände vor, welche dagegen sprechen, dass die amorphen Stoffe etwas anderes sind, als tief unterkühlte Flüssigkeiten.

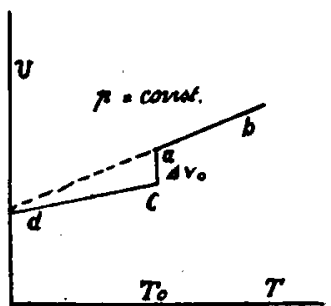

Fig. 139.
Vergleichen wir nun die Volumina eines Stoffes im kristallisierten und im isotropen Zustande bei konstantem Druck $p$ = I Atm. Das Diagramm Fig. I 39 gibt die Volumenisobare des Kristalls, $d c$, und die der leicht beweglichen Flussigkeit, $a b$. Die Verlängerung von $a b$ zu niedrigeren Temperaturen, die gestrichelte Gerade $d a$, gibt dann die Volumina der unterkühlten Flüssigkeit und bei tieferen Temperaturen die Volumina des amorphen Stoffes.

Fur leicht schmelzbare Stoffe gilt nun eine einfache Regel. Die beiden Geraden, welche die Abhängigkeit der Volumina des Stoffes im isotropen und im anisotropen Zustande darstellen, sind so gerichtet, als ob sie sich beim absoluten Nullpunkt der Temperatur schneiden würden. Es gilt nämlich, wenn $\frac{d p V^{\prime}}{d T}$ und $\frac{d p V " *}{d T}$ die Temperaturkoëffizienten des spezifischen Volumens der Flüssigkeit und des Kristalls und $\Delta V_{o}$ die Differenz der spezifischen Volumina beim Schmelzpunkt $T_{o}$ bezeichnen, die Beziehung:

$$
\Delta V_{o}=T_{o}\left(\frac{d p V^{\prime}}{d T}-\frac{d p V^{\prime \prime}}{d T}\right)
$$

Bei hoch schmelzenden Stoffen liegen aber die Verhältnisse anders; bei diesen hat man zwei Falle zu unterscheiden:

I. Das Volumen des Glases ist auch bei den tiefsten Temperaturen grosser als das des
Kristalls. Es scheint das bei allen Silikaten der Fall zu sein.

2. Die beiden Volumenisobaren schneiden sich oberhalb des absoluten Nullpunktes der Temperatur. Ein solcher Fall wurde beim Strontiummetaborat $\left[\mathrm{Sr}\left(\mathrm{BO}_{2}\right)_{2}\right]$ aufgefunden und näher untersucht. Es ergab sich bei $20^{\circ}$ das spezifische Volumen des amorphen Strontiumborates zu $0,3073 \mathrm{ccm}$ pro Gramm und das des kristallisierten $\mathrm{zu} 0,3 \mathrm{I} 84 \mathrm{ccm}$ pro Gramm. Anderseits konnte nachgewiesen werden, dass das Strontiummetaborat bei I I oo ${ }^{n}$ unter Volumenvergrösserung schmilzt, diese Volumenvergrősserung beträgt ungefähr o,02 $\mathrm{ccm}$ pro Gramm Aus der Volumendifferenz des Isotropen und Anisotropen bei $20^{0}$ und der Volumendifferenz bei 1 I $00^{0}$ interpoliert sich die Temperatur, bei der ihre Volumina einander gleich werden, zu $360^{0}$, wăhrend aus den Bestimmungen der Ausdehnungskoëffizienten zwischen 20 und $100^{\circ}$ diese Temperatur sich $z \mathrm{u} 420^{\circ}$ ergibt.

Die Tatsache, dass die Volumenisobaren des Isotropen und Anisotropen sich schneiden konnen, beweist, dass man kontinuierliche Volumenănderungen beim Uebergange eines dieser Zustände in den anderen auf einer kontinuierlich verlaufenden Isobare, wie solche von J. Thomson und van der Waals far die Volumenänderung bei der Verdampfung angenommen wurden, nicht annehmen darf.

Vergleicht man den Energieinhalt der Stoffe im isotropen und anisotropen Zustande, so findet man, dass der Energieinhalt im amorphen Zustande grösser ist als im Kristallzustande, und zwar gilt diese Regel far hoch schmelzende Stoffe (Silikate und Borate) für das Temperaturintervall vom Schmelzpunkt bis zum absoluten Nullpunkt der Temperatur. Für die Hälfte der leicht schmelzbaren Stoffe scheint die Regel zu gelten, dass die Differenz der Energieen beider Phasen beim absoluten Nullpunkt der Temperatur verschwindet. Bei diesen Stoffen ist die Beziehung $r=\left(c_{p}{ }^{\prime}-c_{p^{\prime}}{ }^{\prime \prime}\right) T_{o}$, wo $r$ die Schmelzwărme, $c_{p}^{\prime}$ und $c_{p}^{\prime \prime}$ die spezifischen Wärmen der Schmelze und des Kristalls beim Schmelzpunkt $T_{o}$ bedeuten, in der Mehrzahl der Falle angenähert erfallt.

Stoffe, bei denen die Energiedifferenz des Isotropen und Anisotropen bei höheren Temperaturen durch den Nullwert gehen, sind bisher nicht bekannt geworden.

Die spezifische Wärme des Isotropen ist in der Nahe des Schmelzpunktes immer grösser als die des Kristallisierten. Bei hoch schmelzenden Stoffen, den Silikaten und Boraten, werden tief unterhalb des Schmelzpunktes zwischen 20 und 100 ${ }^{\circ}$ die spezifischen Wärmen des Glases und des Kristalles einander nahezu gleich gefunden, nicht selten ubertrifit die spezifische Wärme des Kristalles um einen geringen Betrag die des Glases. 